\title{
ERRATUM
}

Kazuhiro Kushida • Masataka Shiraki •

Toshitaka Nakamura • Hideaki Kishimoto •

Hirotoshi Morii - Kichizo Yamamoto - Kiyoshi Kaneda •

Masao Fukunaga • Tetsuro Inoue •

Mitsuyoshi Nakashima • Hajime Orimo

\section{Alendronate reduced vertebral fracture risk in postmenopausal Japanese women with osteoporosis: a 3-year follow-up study}

\section{J Bone Miner Metab (2004) 22:462-468 DOI 10.1007/s00774-004-0508-0}

Please note that there is an error on p. 464: On line 6 from the bottom of the right column, " 0.42 " should be " 0.41 ".

K. Kushida $(\square)$

Department of Orthopedic Surgery, Hamamatsu University School of Medicine, 1-20-1 Handayama, Hamamatsu 431-3192, Japan

M. Shiraki

Research Institute and Practice for Involutional Diseases, Nagano,

Japan

T. Nakamura

University of Occupational and Environmental Health, Fukuoka,

Japan

H. Kishimoto

Sanin Rosai Hospital, Tottori, Japan

H. Morii

Japan Osteoporosis Society, Osaka, Japan

K. Yamamoto

Hakuai Hospital, Tottori, Japan

K. Kaneda

Bibai Rosai Hospital, Hokkaido, Japan

M. Fukunaga

Kawasaki Medical School, Okayama, Japan

T. Inoue

Aoyama General Hospital, Aichi, Japan

M. Nakashima

Hamamatsu Institute of Clinical Pharmacology and Therapeutics,

Hamamatsu, Japan

H. Orimo

Tokyo Metropolitan Geriatric Medical Center, Tokyo, Japan 